\title{
The Regularization for the Zeta Functions with Physical Applications II
}

\author{
Minoru Fujimoto ${ }^{1}$, Kunihiko Uehara ${ }^{2}$ \\ ${ }^{1}$ Seika Science Research Laboratory, Seika-cho, Kyoto, 619-0237, Japan \\ ${ }^{2}$ Department of Phy sics, Tezukay ama University, Nara, 631-8501, Japan
}

\begin{abstract}
We have proposed a regularization technique and applied it to the Eu ler product of the zeta functions in the part one. In this paper that is the second part of the trilogy, we aim the nature of the non-trivial zero for the Riemann zeta function which gives us another evidence to demonstrate the Riemann hypotheses by way of the approximate functional equation.Some other results on the critical line are presented using the relations between the Euler product and the deformed summation representations in the critical strip. We also discuss a set of equations which yields the primes and the zeros of the zeta functions. In part three, we will focus on physical applications using these outcomes.
\end{abstract}

Keywords ZetaFunction, Riemann Hypothesis, Prime Numbers, Non-Trivial Zeros

\section{Introduction}

In the situation that the regularizations by the zet a function have been successful with some phys ical applications, [2] [5][13,14] we proposed a regularization technique[7] applicable to the Euler product representation and gave an evidence for the Riemann hypotheses by using this technique in the part one. In this part, we now focus on the zeros of the Riemann zeta function and the surrounding properties of the zeros including other evidences to demonstrate the Riemann hypotheses.[1] The Euler product representation, which played an es sential role in the first part, will be interpreted in terms of the summation representation on the critical line $\mathfrak{R} z=1 / 2$.

The definition of the Riemann zeta function is

$$
\zeta(z)=\lim _{n \rightarrow \infty} \zeta_{n}(z)=\lim _{n \rightarrow \infty} \sum_{k=1}^{n} \frac{1}{k^{z}}=\prod_{k=1}^{\infty}\left(1-\frac{1}{p_{k} z}\right)^{-1}
$$

for $\mathfrak{R} z>1$, where the right hand side is the Euler product representation and $p_{k}$ is the $k$-th prime number. Hereafter we adopt a notation $\hat{\zeta}(z)$ for $\mathfrak{R} z>0$ such as

$$
\hat{\zeta}(z)=\lim _{n \rightarrow \infty} \hat{\zeta}_{n}(z)=\lim _{n \rightarrow \infty} \frac{1}{1-2^{1-z}} \sum_{k=1}^{n} \frac{(-1)^{k-1}}{k^{z}},
$$

which is well regularized even in the critical strip $0<\mathfrak{R} z<1$.

Considering the approximate expansion formula for the Riemann zeta function, we propose an evidence for an elegant proof of the Riemann hypothesis in section 2.and in

* Corresponding author:

uehara@tezukayama-u.ac.jp (KunihikoUehara)

Published online at http://journal.sapub.org/ijtmp

Copyright (C 2012 Scientific \& Academic Publishing. All Rights Reserved §3we show surrounding properties of the zeros for the Riemann zeta function by deforming the Euler product representation to the summation form on the critical line. We study the relation between the primes and the zeros of the zeta function in connection with the Sato-Tate conjecture in section 4 , and we will discuss the equations for these primes and zeros in $\S 5$.

\section{The Expansion Formula and the Riemann Hypothesis}

The Eu ler-Maclaurin sum formula is given by

$$
\begin{gathered}
\sum_{n=M}^{N} f(n)=\int_{M}^{N} f(x) d x+\frac{1}{2}(f(M)+f(N)) \\
+\sum_{j=1}^{k} \frac{B_{2 j}}{(2 j) !}\left[f^{(2 j-1)}(x)\right]_{M}^{N}+R_{2 k},
\end{gathered}
$$

where $B_{2 j}$ is the $2 j$-th Bernoulli nu mber and the remainder term:

$$
\begin{aligned}
R_{2 k} & =\frac{1}{(2 k+1) !} \int_{M}^{N} \bar{B}_{2 k+1}(x) f^{(2 k+1)}(x) d x, \\
\bar{B}_{2 k+1}(x) & =B_{2 k+1}(x-[x]) .
\end{aligned}
$$

We parametrize a complex variable $z$ by two real variable such as $z=s(1 / 2+i t)$ as sameas that in the first part. Using the Euler-Maclaurin sum formula on the assumption of $s / 2=\mathfrak{R} z>-2 k$, we get the relation

$$
\begin{aligned}
\sum_{n=M}^{\infty} \frac{1}{n^{z}} & =\int_{M}^{\infty} x^{-z} d x+\frac{1}{2} M^{-z}+\int_{M}^{\infty} \bar{B}_{1}(x)(-z) x^{-z-1} d x \\
& =\frac{M^{1-z}}{z-1}+\frac{1}{2} M^{-z}+\sum_{j=1}^{k} \frac{B_{2 j}}{(2 j) !} M^{1-z-2 j} \prod_{l=0}^{2 j-2}(z+l)+R_{2 k},
\end{aligned}
$$

where

$$
R_{2 k}=\frac{-z(z+1) \cdots(z+2 k)}{(2 k+1) !} \int_{M}^{\infty} \bar{B}_{2 k+1}(x) x^{-z-2 k-1} d x \text {. (6) }
$$


As is well known we can go forward to the expansion formula in the case of $M \geq 2$,

$$
\begin{aligned}
& \hat{\zeta}(z)=\sum_{n=1}^{M-1} \frac{1}{n^{z}}+\frac{M^{1-z}}{z-1}+\frac{1}{2} M^{-z} \\
& +\sum_{j=1}^{k} \frac{B_{2 j}}{(2 j) !} M^{1-z-2 j} \prod_{l=0}^{2 j-2}(z+l)+R_{2 k} .
\end{aligned}
$$

The remainder term $R_{2 k}$ can be estimated as follows:

$$
\begin{aligned}
\left|R_{2 k}\right| & \leq \frac{\pi^{2}}{3}\left|\frac{z+2 k+1}{\frac{1}{2}+2 k+1}\right|\left|\frac{z(z+1) \cdots(z+2 k)}{(2 \pi)^{2 k+2}}\right| M^{-\frac{1}{2}-2 k-1} \\
& \leq C(k) \sqrt{M}\left(\frac{t}{2 \pi M}\right)^{2 k+2},
\end{aligned}
$$

where we put $s=1$ and $C(k)$ is constant only depending on $k$. This tells us that it is necessary for the remainder term to be $M>t /(2 \pi)$ to converge.

Taking account of the Euler-Maclaurin sum formula, we can put the regularized zeta function as

$$
\lim _{n \rightarrow \infty} \hat{\zeta}_{n}(z)=\lim _{n \rightarrow \infty}\left\{\zeta_{n}(z)-\frac{n^{1-z}}{1-z}\right\}
$$

and a zero in the critical strip is the solution to the equation

$$
\hat{\zeta}(z)=\lim _{n \rightarrow \infty} \hat{\zeta}_{n}(z)=0 .
$$

These equations (9) and (10) are identical to the equation (A4) in the first part. As stated in Appendix A in the first part, Eq.(2) can be derived by way of the regularization method developed in the part one, which means that we can reach here besides using the Euler-Maclaurin sum formula. As (1 $-\rho$ ) is also the solution when $\rho$ is the solution of Eq.(10), asolution of the equation

$$
\hat{\zeta}(1-z)=0
$$

is also a zero. Now we transform Eq.(9) to

$$
\lim _{n \rightarrow \infty}\left\{(1-z) \zeta_{n}(z)-n^{1-z}\right\}=0
$$

and substituting $(1-z)$ for $z$, we get

$$
\lim _{n \rightarrow \infty}\left\{z \zeta_{n}(1-z)-n^{z}\right\}=0 .
$$

Combin ing these equations (12) and (13), we get

$$
\lim _{n \rightarrow \infty}\left\{z(1-z) \zeta_{n}(z) \zeta_{n}(1-z)-n\right\}=0,
$$

namely,

$$
z^{2}-z+\lim _{n \rightarrow \infty} \frac{n}{\zeta_{n}(z) \zeta_{n}(1-z)}=0 .
$$

The solution $\rho_{n}$ of the equation $\hat{\zeta}_{n}(z)=0$ is satisfied the relation

$$
\rho_{n}=\frac{1}{2} \pm i \sqrt{\frac{n}{\zeta_{n}\left(\rho_{n}\right) \zeta_{n}\left(1-\rho_{n}\right)}} .
$$

On the other hand, the approximate functional equation by Hardy and Little wood[4], which leads to the Riemann-Siegel formula, is given by

$$
\begin{gathered}
\hat{\zeta}(z)=\sum_{n \leq x} \frac{1}{n^{z}}+\hat{H}(z) \sum_{n \leq y} \frac{1}{n^{1-z}} \\
+O\left(x^{-s / 2}\right)+O\left(|t|^{1 / 2-s / 2} y^{s / 2-1}\right),
\end{gathered}
$$

where $0 \leq s / 2(=\mathfrak{R} z) \leq 1, x \geq 1, y \geq 1,2 \pi x y=|t|$ and $\hat{H}(z)$ is given by

$$
\hat{H}(z)=2 \Gamma(1-z)(2 \pi)^{z-1} \sin \frac{\pi z}{2} .
$$

For $s>2$, the relation

$$
\zeta(z)=H(z) \sum_{n=1}^{\infty} \frac{1}{n^{1-z}}=H(z) \zeta(1-z)
$$

is satisfied and we write down $\hat{\zeta}(1-z)$ for $0<\mathfrak{R} z<1$ as,

$$
\hat{\zeta}(1-z)=\frac{1}{1-2^{z}} \sum_{n=1}^{\infty} \frac{(-1)^{n-1}}{n^{1-z}} .
$$

Then the relation

$$
\hat{\zeta}(z)=\hat{H}(z) \hat{\zeta}(1-z)
$$

is satisfied for $\mathfrak{R} z 0$ and substituting $(1-z)$ for $z$, we conclude Eq.(21) is satis fied for all $z$.

We set $n \leq x, n \leq y$ and $|t|=2 \pi x y \geq 2 \pi n^{2}>2 \pi n$, then

$$
\hat{\zeta}(z)=\zeta_{n}(z)+\hat{H}(z) \zeta_{n}(1-z)+R_{n}(z),
$$

where the remainder term $R_{n}(z)$ :

$$
\begin{aligned}
R_{n}(z) & =O\left(n^{-s / 2}\right)+O\left(|t|^{1 / 2-s / 2} n^{s / 2-1}\right) \\
& =O\left(n^{-s / 2}\right)+o\left((2 \pi n)^{1 / 2-s / 2} n^{s / 2-1}\right)
\end{aligned}
$$

can be ignored by taking the limit of $n \rightarrow \infty$, namely

$$
\lim _{n \rightarrow \infty} R_{n}(z)=\lim _{n \rightarrow \infty}\left\{O\left(n^{-s / 2}\right)+o\left(n^{-1 / 2}\right)\right\}=0 .
$$

We put the relations at zeros

$$
\zeta_{n}\left(\rho_{n}\right)=\frac{n^{1-\rho_{n}}}{1-\rho_{n}}, \quad \zeta_{n}\left(1-\rho_{n}\right)=\frac{n^{\rho_{n}}}{\rho_{n}}
$$

into Eq.(22) together with $\hat{\zeta}_{n}\left(\rho_{n}\right)=0$, we can write

$$
\begin{aligned}
\frac{n^{1-\rho_{n}}}{1-\rho_{n}}+\hat{H}\left(\rho_{n}\right) \frac{n^{\rho_{n}}}{\rho_{n}}+R_{n}\left(\rho_{n}\right) & =0, \\
\frac{n^{1-2 \rho_{n}}}{1-\rho_{n}}+\hat{H}\left(\rho_{n}\right) \frac{1}{\rho_{n}}+R_{n}\left(\rho_{n}\right) \frac{1}{n^{\rho_{n}}} & =0 .
\end{aligned}
$$

Taking the limit of $\mathrm{n} \rightarrow \infty$, we get the relations

$$
\lim _{n \rightarrow \infty}\left|\frac{n^{1-2 \rho_{n}}}{1-\rho_{n}}\right|=-\lim _{n \rightarrow \infty}\left|\frac{\hat{H}\left(\rho_{n}\right)}{\rho_{n}}\right|=-\left|\frac{\hat{H}(\rho)}{\rho}\right| .
$$

The right hand side of Eq.(27) is finite, so the numerator of the left hand side $\lim _{n \rightarrow \infty}\left|n^{1-2 \rho_{n}}\right|$ must be finite. This means that the real part of $\left(1-2 \rho_{n}\right)$ must converge to 0 in the limit of $\mathrm{n} \rightarrow \infty$ when the real part of $\left(1-2 \rho_{n}\right)$ is positive. When $\mathfrak{R}\left(1-2 \rho_{n}\right)<0$, we re write the left hand side of Eq.(27) like

$$
\lim _{n \rightarrow \infty} \frac{n^{1-2\left(1-\rho_{n}\right)}}{1-\left(1-\rho_{n}\right)}=\lim _{n \rightarrow \infty} \frac{n^{2 \rho_{n}-1}}{\rho_{n}},
$$

so we get the same goal as $\lim _{n \rightarrow \infty} \mathfrak{R}\left(2 \rho_{n}-1\right)=0$. After all, the Riemann hypothesis is satisfied including the trivial case $\mathfrak{R}\left(2 \rho_{n}-1\right)=0$,

$$
\rho=\lim _{n \rightarrow \infty} \rho_{n}=\lim _{n \rightarrow \infty}\left(\frac{1}{2}+i t_{n}\right)=\frac{1}{2}+i \lambda,
$$

where $\lambda$ is real but $t_{n}$ is not necessarily a real number.[8]

Now we think about the values of $t_{n}$, which converge to a positive $\lambda$ in the limit of $n \rightarrow \infty$ and put it into Eq.(16)

$$
\begin{aligned}
\rho_{n} & =\frac{1}{2}+i \sqrt{\frac{n}{\zeta_{n}\left(\rho_{n}\right) \zeta_{n}\left(1-\rho_{n}\right)}} \\
& =\frac{1}{2}+i \sqrt{\frac{n}{\zeta_{n}\left(\frac{1}{2}+i t_{n}\right) \zeta_{n}\left(\frac{1}{2}-i t_{n}\right)}} .
\end{aligned}
$$

Thus we write the solution to the equation $\hat{\zeta}(z)=0$ 


$$
\begin{gathered}
\rho=\lim _{n \rightarrow \infty}\left\{\frac{1}{2}+i \sqrt{\frac{n}{\zeta_{n}\left(\rho_{n}\right) \zeta_{n}\left(1-\rho_{n}\right)}}\right\} \\
\text { i.e. } \\
\lambda=\lim _{n \rightarrow \infty} \sqrt{\frac{n}{\zeta_{n}\left(\frac{1}{2}+i t_{n}\right) \zeta_{n}\left(\frac{1}{2}-i t_{n}\right)}} .
\end{gathered}
$$

Using the $n$-th order relation of Eq.(21)

$$
\zeta_{n}\left(\frac{1}{2}-i t_{n}\right)=H\left(\frac{1}{2}-i t_{n}\right) \zeta_{n}\left(\frac{1}{2}+i t_{n}\right),
$$

we get the relation

$$
\begin{aligned}
t_{n} & =\sqrt{\frac{n}{\zeta_{n}\left(\frac{1}{2}+i t_{n}\right) H\left(\frac{1}{2}-i t_{n}\right) \zeta_{n}\left(\frac{1}{2}+i t_{n}\right)}} \\
& =\frac{1}{\zeta_{n}\left(\frac{1}{2}+i t_{n}\right)} \sqrt{\frac{n}{H\left(\frac{1}{2}-i t_{n}\right)}} .
\end{aligned}
$$

This form will be utilize to calculate zeros of the Riemann zeta function by way of the limit of $n \rightarrow \infty$.

\section{The Euler Product and a Summation Representation}

We write down the Euler product representation for the standard form described as same as the equation (25) in the first part

$$
\begin{gathered}
\frac{1}{\left|\zeta_{n}\left(s\left(\frac{1}{2}+i t\right)\right)\right|^{2}}=f_{n}(s, t) \\
=\prod_{k=1}^{n}\left(1-\frac{2}{p_{k}^{s / 2}} \cos \left(s t \log p_{k}\right)+\frac{1}{p_{k} s}\right) .
\end{gathered}
$$

Here $f_{n}(s, t)$ and $\log f_{n}(s, t)$ will diverge at the same time in $n \rightarrow \infty$, because $f_{n}(s, t)$ is positive. As all non-trivial zeros of the Riemann zeta function is expected on $s=1$, we study the following relation for $s \geq 1$

$$
\begin{gathered}
=\sum_{k=1}^{n} \log \left(1-\frac{2}{p_{k}^{s / 2}} \cos \left(s t \log p_{k}\right)+\frac{1}{p_{k}^{s}}\right) \\
=\sum_{k=1}^{n} \log \left\{1-\left(\frac{2}{p_{k}^{s / 2}} \cos \left(s t \log p_{k}\right)-\frac{1}{p_{k}{ }^{s}}\right)\right\} \\
=-\sum_{k=1}^{n} \sum_{m=1}^{\infty} \frac{1}{m}\left(\frac{2}{p_{k}^{s / 2}} \cos \left(s t \log p_{k}\right)-\frac{1}{p_{k}^{s}}\right)^{m} \\
=-\sum_{k=1}^{n}\left(\frac{2}{p_{k}^{s / 2}} \cos \left(s t \log p_{k}\right)-\frac{1}{p_{k}^{s}}\right)-\frac{1}{2} \sum_{k=1}^{n}\left(\frac{2}{p_{k}^{s / 2}} \cos \left(s t \log p_{k}\right)-\frac{1}{p_{k}^{s}}\right)^{2} \\
-\frac{1}{3} \sum_{k=1}^{n}\left(\frac{2}{p_{k}^{s / 2}} \cos \left(s t \log p_{k}\right)-\frac{1}{p_{k}^{s}}\right)^{3}+(\text { finite terms for } n \rightarrow \infty) .
\end{gathered}
$$

We regularize Eq.(35) in order to apply it even in the case $s=1$. We try to regularize Eq.(35) by way of dividing an appropriate factor $\sum_{k=1}^{n} p_{k}^{-1}$, which leaves a leading divergence divergent and makes a non-leading divergence convergent. In fact, we divide Eq.(35) by

$$
\prod_{k=1}^{n}\left(1+\frac{1}{p_{k}}\right)
$$

which we also adopted in the equation (26) of the first part.[7] Thus we study the divergence in the form of

$$
\frac{\prod_{k=1}^{n}\left(1-\frac{2}{p_{k}^{s / 2}} \cos \left(s t \log p_{k}\right)+\frac{1}{p_{k}^{s}}\right)}{\prod_{k=1}^{n}\left(1+\frac{1}{p_{k}}\right)}
$$

which corresponds in the summation form as

$$
\frac{-2 \sum_{k=1}^{n} \frac{\cos \left(t \log p_{k}\right)}{\sqrt{p_{k}}}\left(\frac{\cos \left(t \log p_{k}\right)}{\sqrt{p_{k}}}+1\right)+\sum_{k=1}^{n} \frac{1}{p_{k}}}{\sum_{k=1}^{n} \frac{1}{p_{k}}},
$$

where we set $s=1$. The leading divergent term of Eq.(38) in $n \rightarrow \infty$ is

$$
\sum_{k=1}^{n} \frac{\cos \left(t \log p_{k}\right)}{\sqrt{p_{k}}}
$$

The form of divisor $\prod_{k=1}^{n}\left(1+p_{k}^{-1}\right)$ means that using Mertens' theorem

$$
\prod_{k=1}^{m}\left(1-\frac{1}{p_{k}}\right)=\frac{e^{-\gamma}}{\log p_{m}}\left(1+O\left(\frac{1}{\sqrt{p_{m}}}\right)\right)
$$

and the Euler's $\zeta(2)$

$$
\prod_{k=1}^{\infty}\left(1-\frac{1}{p_{k}^{2}}\right)=\frac{6}{\pi^{2}}
$$

we get

$$
\begin{aligned}
C \prod_{k=1}^{m}\left(1+\frac{1}{p_{k}}\right) & =\prod_{k=1}^{m} \frac{\left(1-\frac{1}{p_{k}^{2}}\right)}{\left(1-\frac{1}{p_{k}}\right)} \prod_{k=m+1}^{\infty}\left(1-\frac{1}{p_{k}^{2}}\right) \\
& =\frac{6 e^{\gamma}}{\pi^{2}} \log p_{m}\left(1+O\left(\frac{1}{\sqrt{p_{m}}}\right)\right), \\
\log \prod_{k=1}^{m}\left(1+\frac{1}{p_{k}}\right) & =\sum_{k=1}^{m} \log \left(1+\frac{1}{p_{k}}\right) \\
& =\sum_{k=1}^{m} \frac{1}{p_{k}}-\frac{1}{2}\left(\sum_{k=1}^{m} \frac{1}{p_{k}}\right)^{2}+\cdots \\
& \simeq \log \log p_{m},
\end{aligned}
$$

where

$$
\frac{6 e^{\gamma}}{\pi^{2}} \leq C \leq e^{\gamma}
$$

The Euler product representation for $n \rightarrow \infty$ is only valid for $s \geq 2$, and we restrict our interest for $t>0$. The zeros of the Riemann zeta function make Eq.(34) divergent, which means that products are multiplied maximally in the right hand side. Each term in Eq. (34) is maximized when $\cos \left(s t \log p_{k}\right)=-1$, namely, $s t=(2 \ell-1) \pi / \log p_{k}(\ell=$ natural number $)$. We give graphs for the superposition of cosine functions, which indicate the solution of $\cos \left(t \log p_{k}\right)=-1$ as local maximum values,

$$
y_{n, \alpha}(t)=-\sum_{k=1}^{n} \frac{\cos \left(t \log p_{k}\right)}{p_{k}{ }^{\alpha}} .
$$

The graph of $y_{n \times \alpha}(t)$ for $\alpha=1 / 2$ is printed as Figure 1, and judging from the graph of $\alpha=1$ (Figure 2), the denominator $p_{k}$ seems to be well-matched to cancel the notches come from the superposition of cosine functions. Figure 1 is also such an example of notches. Figure 2 has the positive maximal values that correspond to the non-trivial zeros of the zeta function except the one appeared in $t<6$. Thus zeros of the 
Euler product representation in Eq.(34) preserve the value even in the form of the summation in Eq.(45).

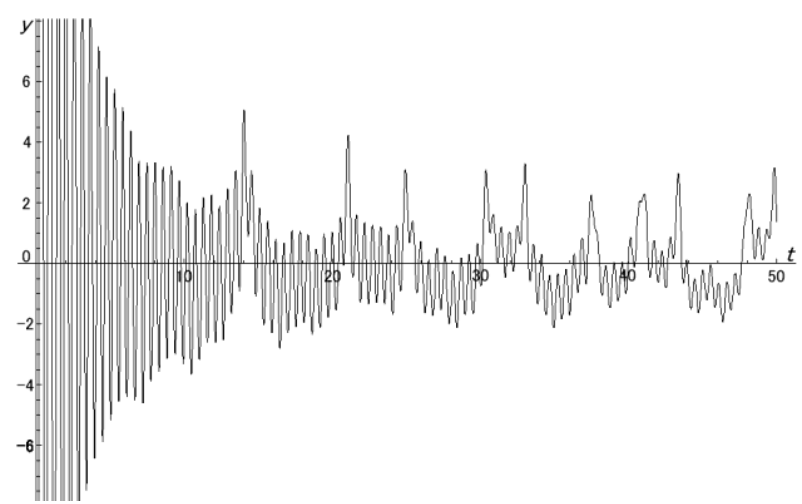

Figure 1. The graph of $y_{n, a}$ for $n=10^{4}$ and $\alpha=1 / 2$

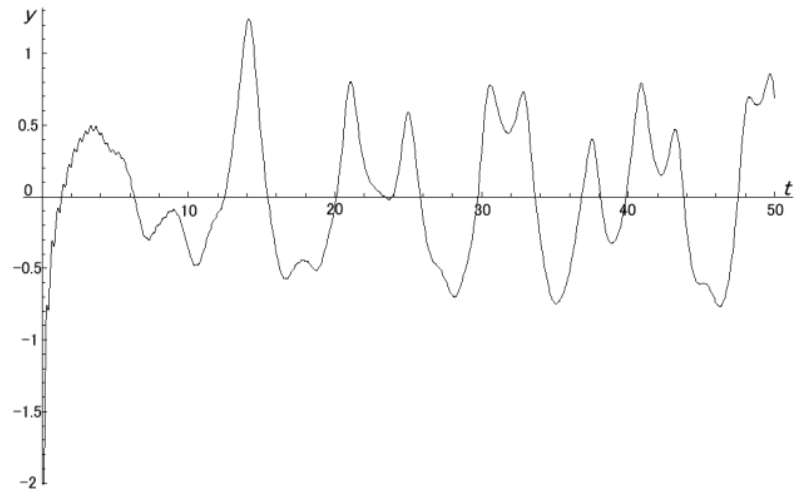

Figure 2. The graph of $y_{n, a}$ for $n=10^{6}$ and $\alpha=1$

The terms to regularize the divergence will be discussed, which is essential to the order on the critical line and seems to be closely related to the von Mangoldt function, [9] in a separate paper.

On the other hand, the sum over the zeros of the zeta function for a certain prime $p$

$$
-2 \sum_{j=1}^{n} \sqrt{p} \cos \left(\lambda_{j} \log p\right)
$$

leads us a graph which indicates locations of the prime numbers.[3]
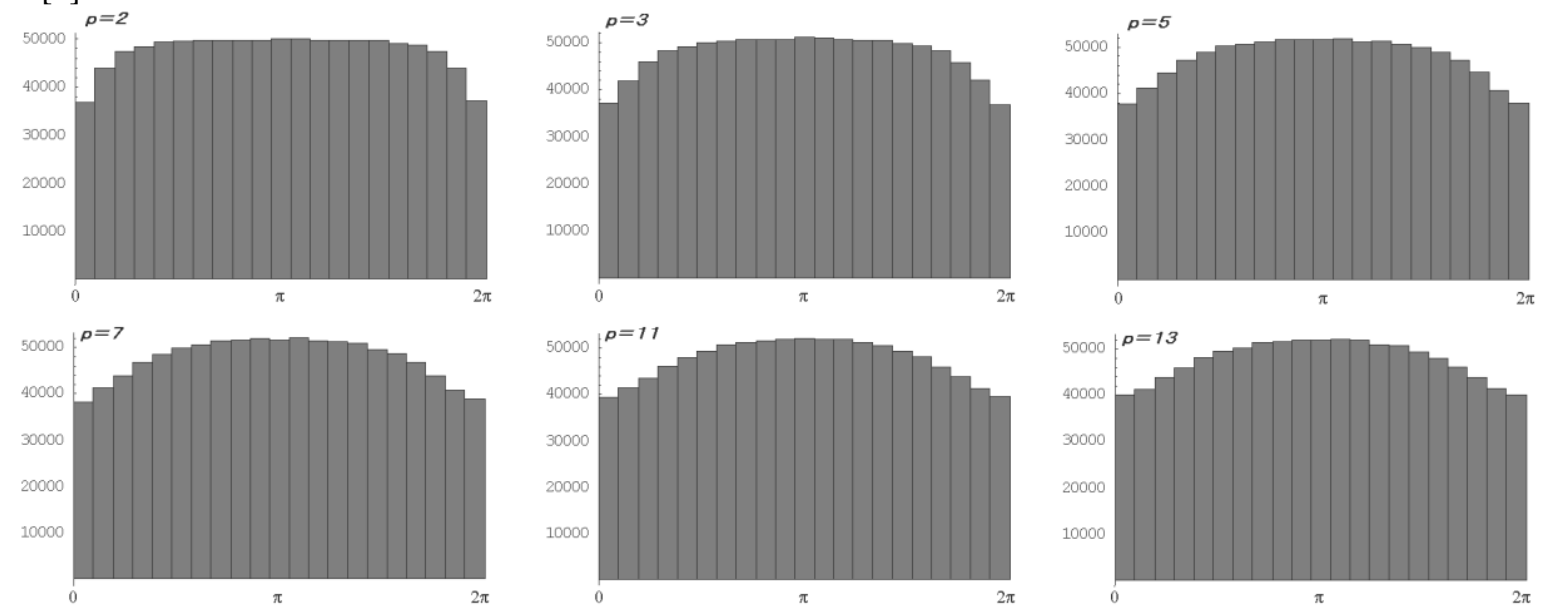

Figure 3. The hist ograms(divided $21 \mathrm{st}$ ) of distributions for $p_{k}=2,3,5,7,11$ and 13 beginning with $j=10^{6}$ up to $2 \times 10^{6}$ 

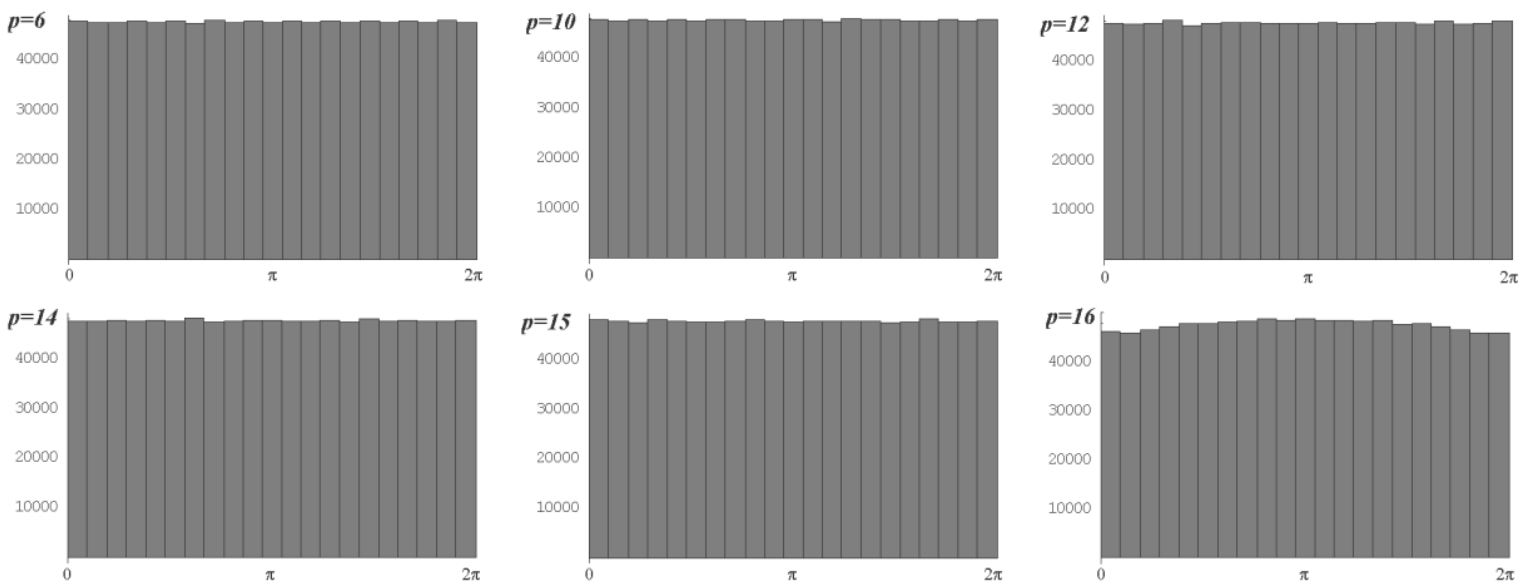

Figure 4. The histograms(divided $21 \mathrm{st}$ ) of distributions for $p=6,10,12,14,15$ and 16 beginning with $j=10^{6}$ up to $2 \times 10^{6}$

$$
\frac{2}{\pi} \int_{\alpha}^{\beta} \sin ^{2} \theta d \theta
$$

where $0 \leq \alpha \leq \theta \leq \beta \leq \pi$. On the other hand, once we put $2 \theta=\lambda_{j} \log p_{k}$, we may claim that the response of $j$-direction increase of $\lambda_{j}$ yields the distribution of the Wigner's semi-circle law, which is related by regarding $\cos \theta$ of Eq.(47) as a single variable.

Figure 3 is the histograms of distributions for $p_{k}=$ $2,3,5,7,11$ and 13. In contrast to these histograms, the histograms of distributions in case that we put composite numbers $(=6,10,12,14,15$ and 16$)$ into $p_{k}$, are also printed in Figure 4. In the cases for the power of one prime like $p=16$, a shape of the peak around $\pi$ slightly remains in the histogram, whereas the shape of the tales near 0 or $2 \pi$ would be convex down wards.

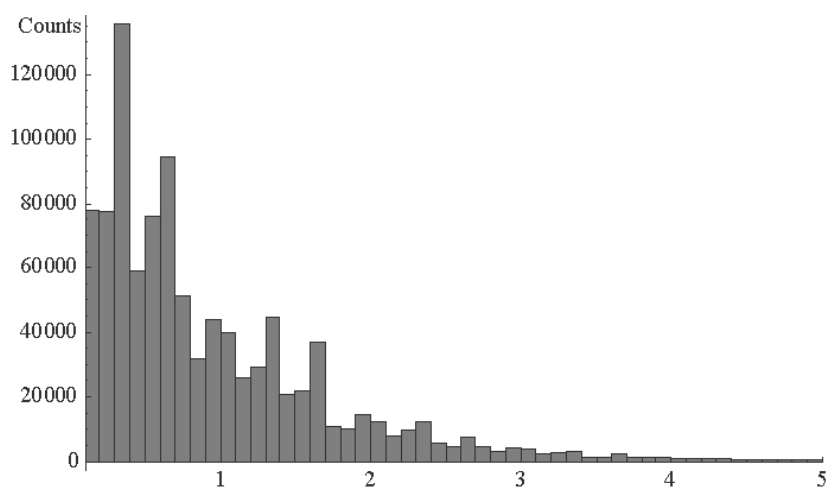

Figure 5. The histogram(class interval $=0.1$ ) for the distribution of the interval of $p_{k} / \log p_{k}$ for $10^{6}$ primes beginning with $k=10^{6}$

A nature of primes is also found in a distribution for the interval of succeeding primes,

$$
\frac{p_{k+1}}{\log p_{k+1}}-\frac{p_{k}}{\log p_{k}}
$$

where the logarithm terms exist in order to normalize to one. We present the histogram for $10^{6}$ primes beginning with $k=$ $10^{6}$ in Figure 5 for example. The fluctuation in the histogram which rather looks like an oscillation never vanish for larger number of primes and is deeply related to the Wilson theorem and the Hoheisel's constant.[10]

\section{Discussions and Remarks}

We discuss the equations which yield the primes and the zeros of the zeta functions in this section. We normalize the product of $\lambda_{j}$ and $\log p_{k}$ introducing new notations $\mu_{j}$ and $v_{k}$ as

$$
\mu_{j}=\lambda_{j}, \quad \nu_{k}=\frac{\log p_{k}}{2 \pi},
$$

and the $k$-direction $(k=1,2, \cdots, \infty)$ average of

$\mu_{j} v_{k}-1 / 2-\left[\mu_{j} v_{k}\right]$ will be 0 by the distribution like the Sato-Tate conjecture, where[ ] is the Gauss symbol. By the law of large number, we can write down

$$
\sum_{k=1}^{m}\left(\mu_{j} \nu_{k}-\frac{1}{2}\right)-\sum_{k=1}^{m}\left[\mu_{j} \nu_{k}\right]=O\left(\frac{1}{m}\right),
$$

so we get

$$
\mu_{j}=\frac{1}{\sum_{k=1}^{m} \nu_{k}}\left\{\sum_{k=1}^{m}\left[\mu_{j} \nu_{k}\right]+\frac{m}{2}+O\left(\frac{1}{m}\right)\right\} .
$$

We can estimate the denominator as [15]

$$
\begin{aligned}
\log p_{k} & =\log (k \log k+O(\log k)) \\
& =\log k+O(\log \log k), \\
\nu_{k} & =\frac{1}{2 \pi} \log p_{k}=\frac{1}{2 \pi}(\log k+O(\log \log k)), \\
\sum_{k=1}^{m} \nu_{k} & =\sum_{k=1}^{m} \frac{1}{2 \pi} \log p_{k} \\
& \simeq \frac{1}{2 \pi} \int_{1}^{m}(\log x+O(\log \log x)) d x \\
& \simeq \frac{1}{2 \pi} m(\log m-1)+O((\log \log m) \log m) .
\end{aligned}
$$

After all, we write a following approximate relation for any $\mu_{i}$,

$$
\begin{aligned}
\mu_{j} & =\frac{\sum_{k=1}^{m}\left[\mu_{j} \nu_{k}\right]+\frac{m}{2}+O\left(\frac{1}{m}\right)}{\frac{1}{2 \pi} m(\log m-1)+O((\log \log m) \log m)} \\
& =\frac{2 \pi \sum_{k=1}^{m}\left[\mu_{j} \nu_{k}\right]}{m(\log m-1)}+\frac{\pi}{\log m-1}+O\left(\frac{1}{m^{2} \log m}\right),
\end{aligned}
$$

using $\lambda_{j}=\mu_{j}, v_{k}=\log p_{k} /(2 \pi)$,we write the relation for any $\lambda_{j}$ 


$$
\lambda_{j}=\frac{2 \pi \sum_{k=1}^{m}\left[\frac{\lambda_{j} \log p_{k}}{2 \pi}\right]}{m(\log m-1)}+\frac{\pi}{\log m-1}+O\left(\frac{1}{m^{2} \log m}\right) .
$$

In the similar way,[12] we take the $j$-direction average of $\mu_{j} v_{k}-1 / 2-\left[\mu_{j} v_{k}\right]$, we can writedown by a symmetric property as illustrated in Figure 3, we get

$$
\nu_{k}=\frac{1}{\sum_{j=1}^{n} \mu_{j}}\left\{\frac{n}{2}+\sum_{j=1}^{n}\left[\mu_{j} \nu_{k}\right]+O\left(\frac{1}{n}\right)\right\} .
$$

We also estimate the denominator as

$$
\begin{aligned}
\lambda_{j} & =\frac{2 \pi j}{\log j+\log 2 \pi}+O\left(\frac{j}{\log ^{2} j}\right) \\
\sum_{j=1}^{n} \lambda_{j} & =\lambda_{1}+2 \pi \sum_{j=2}^{n} \frac{j}{\log j}+\sum_{j=2}^{n} O\left(\frac{j}{\log ^{2} j}\right) \\
& =2 \pi \int_{2}^{n} \frac{x}{\log x} d x+\int_{2}^{n} O\left(\frac{x}{\log ^{2} x}\right) d x \\
& =2 \pi\left[\frac{x^{2}}{2 \log x}\right]_{2}^{n}+\pi \int_{2}^{n} \frac{x}{\log ^{2} x} d x+\left[O\left(\frac{j}{\log ^{2} j}\right)\right]_{2}^{n}( \\
& =\frac{\pi n^{2}}{\log n}+O\left(\frac{n^{2}}{\log ^{2} n}\right)=\sum_{j=1}^{n} \mu_{j}
\end{aligned}
$$

$$
=\frac{1}{2 \pi}\left(\frac{2 \log n \sum_{j=1}^{n}\left[\mu_{j} \nu_{k}\right]}{n^{2}}+\frac{\log n}{n}\right)+O\left(\frac{\log n}{n^{3}}\right) .
$$

Finally we write down the relation for any $p_{k}$

$$
\begin{aligned}
&= \frac{\log p_{k}=2 \pi \nu_{k}}{\log \sum_{j=1}^{n}\left[\frac{\lambda_{j} \log p_{k}}{2 \pi}\right]} n^{2}+\frac{\log n}{n}+O\left(\frac{\log n}{n^{3}}\right) \\
& p_{k}=\exp \left\{\frac{2 \log n \sum_{j=1}^{n}\left[\frac{\lambda_{j} \log p_{k}}{2 \pi}\right]}{n^{2}}+\frac{\log n}{n}\right\} \cdot \exp \left\{O\left(\frac{\log n}{n^{3}}\right)\right\}_{(62)} \\
&=n
\end{aligned}
$$

Equations (56) and (62) are a set of equations which gives prime numbers and zeros of the Riemann zeta function.

\section{REFERENCES}

[1] P. Borwein, S. Choi, B. Rooney and A. Weirathmueller,The Riemann Hypothesis: A Resource for the Afficionado and Virtuoso Alike, CMS Books inMathematics, SpringerVerlag, 2008.

[2] A. Connes and M. Marcolli, NoncommutativeGeometry, Quantum Fields and Motives, AMS, 2008.

[3] J. B. Conrey, Figure 6 in "The Riemann Hypothesis, Notice of the AMS,pp.341-353, 2003".

[4] J. B. Conrey, D. W. Farmer, J. P. Keating, M. O. Rubinstein and N. C. Snaith, Integral moments of L-functions, Proceedings of the London Mathematical Society 91, Cambridge University Press, pp.33-104, 2005.

[5] C. P. Dettmann, New horizons in multidimensional diffusion: The Lorentz gas and the Riemann hypothesis. J.Stat. Phys. 146, pp.181-204, 2012

[6] P. D. T. A. Elliot, The Riemann zeta function and coin tossing, J. ReineAngew.Math. 254,pp.100-109, 1972.

[7] M. Fujimoto and K. Uehara, Regularization for zeta functions with phy sical applications I, arXiv:math-ph/0609013, 2006.

[8] M. Fujimoto and K. Uehara, A Brief Note on the Riemann Hypothesis I, II, arXiv:math-ph/0906.1099v2, 1003.2854, 2011.

[9] A. Gut, Some remarks on the Riemann zeta distribution, Uppsala University Department of Mathematics Report, 2005.

[10] G. Hoheisel, Primzahlprobleme in der Analysis, Berliner Sitzungsberichte, pp.580-588, 1930.

[11] B. Mazur, Controlling our errors, Nature 443,pp.38-40, 2006.

[12] A. Odlyzko, The 1020 -th Zero of the Riemann Zeta Function and 70 Million of the Neighbors, AT\&T Bell Lab. Preprint, 1989.

[13] D. Schumayer and D.A.W. Hutchinson, Colloquium: Phy sics of the Riemann hy pothesis. Rev. Mod. Phys. 83, pp.307-330, 2011.

[14] G. Sierra, A physics pathway to the Riemann hypothesis, arXiv:math-ph/1012.4264, 2010.

[15] H. M. Srivastava and J.Choi, Series Associated with the Zeta and Related Function, Kluwer, 2001.

[16] C. J.Swierczewski, Connections Between the Riemann Hypothesis and the Sato-Tate Conjecture, http//modular. math.washington.edu/projects/swierczewski.pdf, 2008. 